\title{
FAMILY LINKS BETWEEN PLANETARY NEBULAE NUCLEI AND CATACLYSMIC VARIABLES, BINARY WHITE DWARFS, R CRB STARS, AND SNE IA
}

\author{
L.R. YUNGELSON AND A.V. TUTUKOV \\ Astronomical Institute of the Russian Academy of Sciences \\ 48 Pyatnitskaya Str., 109017 Moscow, Russia
}

\begin{abstract}
We analyse the population of $\mathrm{PNe}$ and links between binary $\mathrm{PNNi}$ and stars which are in the later evolutionary stages. In a model which assumes that all stars are born in binaries, about $16 \%$ of PN result from ejection of common envelopes in close binaries, $85 \%$ of single PNNi are formed by merger of components of binaries. In the model, $5 \%$ of PNNi may be precataclysmic binaries, $5 \%$ may be precursors of symbiotic stars, $0.4 \%$ may be pre-SN Ia, $0.1 \%$ - precursors of hydrogen-deficient giants. About $0.1 \%$ of all $\mathrm{PNe}$ may be hydrogen-deficient.
\end{abstract}

\section{Introduction}

It is commonly accepted that planetary nebulae $(\mathrm{PNe})$ are produced by ejection of envelopes by AGB stars or by ejection of common envelopes (CEs) by close binaries. Below, we discuss the relative significance of two formation mechanisms for the total population of $\mathrm{PNe}$ and the origin of several interesting astrophysical objects, formation of which may be preceded by the ejection of a PN. Among these objects are cataclysmic variables (CVs), supernovae of type Ia (SNe Ia), hydrogen-deficient (super)giants, and still hypothetical Thorne-Żytkow objects (TŻOs). As PNe formation we consider events in which a hot $\left(T_{e} \geq 30,000 \mathrm{~K}\right)$ and luminous $\left(L \geq 10^{3} L_{\odot}\right)$ object surrounded by an ejected envelope is formed.

We assume that all stars are born in binaries with initial separations $10 \lesssim A_{0} / R_{\odot} \leq 10^{6}$ and follow, by means of a population synthesis code, all evolutionary scenarii for them depending on initial masses of components $M_{10}, M_{20}$, and $A_{0}$. For an initial percentage of binaries different from $100 \%$, all results given below may be rescaled. It is assumed that one binary with 


\section{L.R. Yungelson}

TABLE 1. Birthrates of PNNi in wide and close systems $\left(\mathrm{yr}^{-1}\right)$

\begin{tabular}{lccccccccc}
\hline & WIDE SYSTEMS & \multicolumn{4}{c}{ CLOSE SYSTEMS } \\
\hline Nebula & \multicolumn{3}{c}{ HYDROGEN } & \multicolumn{4}{c}{ HYDROGEN } & \multicolumn{3}{c}{ HELIUM } \\
Companion & - & MS & WD & - & MS & WD & NS & WD & NS \\
Birthrate & 0.02 & 0.52 & 0.006 & 0.11 & 0.10 & 0.01 & 0.0001 & 0.0008 & 0.0003 \\
\hline
\end{tabular}

$M_{10} \geq 0.8 M_{\odot}$ is formed annually in the Galaxy and that star formation rate was constant for $15 \mathrm{Gyr}$. Initially close binaries constitute $40 \%$ of the total sample. This model is appropriate for the Galactic disk. All additional details of the model and population synthesis code may be found in Yungelson et al. $(1993,1995,1996)$. The models of PNe populations formed via ejection of CEs were previously discussed e.g. by de Kool (1990), Iben \& Tutukov (1993), Yungelson et al. (1993), Han et al. (1995).

\section{Results and discussion}

Table 1 summarizes the birthrates of PNNi in wide and close binaries and classifies them by the chemical composition of PN and the nature of companion. The flow chart for scenarios of formation of PNNi and some objects which pass through the PN stage is given below. We use the following abbreviations: MS - main-sequence star, G - giant, PNN - planetary nebula nucleus, WD - carbon-oxygen white dwarf (if the latter is composed of helium a prefix He is used), $\mathrm{CE}$ - common envelope, CV - cataclysmic variable, $\mathrm{SN}$ - supernova, RLOF - steady Roche lobe overflow, $\mathrm{R} \mathrm{CrB} \mathrm{-} \mathrm{hy-}$ drogen deficient giant (which isn't necessarily strictly of an $\mathrm{R} C r B$ variety), HeS - helium star, NS - neutron star, TŻ - Thorne-Żytkow object. Common envelopes and PNe expected to be He-rich are marked by adding ( $\mathrm{He})$.

A. $M S+M S \rightarrow G+M S \rightarrow \mathbf{P N N}+\mathbf{M S} \rightarrow W D+M S \rightarrow W D+G \rightarrow \mathbf{W D}+\mathbf{P N N} \rightarrow W D+W D$

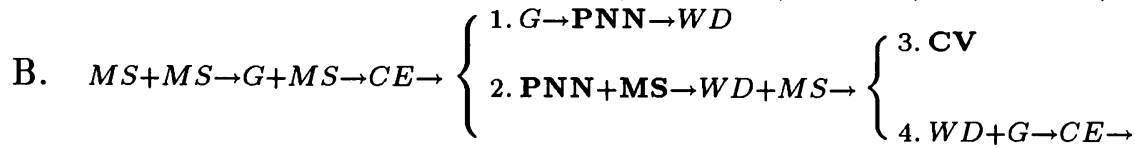

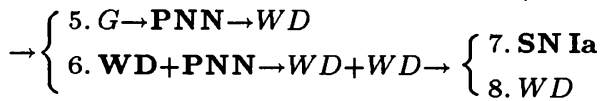

C. $M S+M S \rightarrow R L O F \rightarrow H e W D+M S \rightarrow H e W D+G \rightarrow C E \rightarrow$ HeWD+PNN $\rightarrow$

$$
\rightarrow H e W D+W D \rightarrow \mathbf{R} \mathbf{C r B} \rightarrow \mathbf{P N N}(\mathbf{H e}) \rightarrow W D
$$

D. $M S+M S \rightarrow G+M S \rightarrow C E \rightarrow H e S+M S \rightarrow C E(H e) \rightarrow\left\{\begin{array}{l}1 . \mathrm{PNN}(\mathrm{He})+\mathrm{MS} \rightarrow \text { as in B.2 } \\ 2 . G \rightarrow \mathbf{P N N} \rightarrow W D\end{array}\right.$

E. $M S+M S \rightarrow G+M S \rightarrow C E \rightarrow H e S+M S \rightarrow S N I b+M S \rightarrow N S+M S \rightarrow N S+G \rightarrow C E \rightarrow$

$\rightarrow N S+H e S \rightarrow C E(H e) \rightarrow \mathbf{N S}+\mathbf{P N N}(\mathbf{H e}) \rightarrow N S+W D \rightarrow \mathbf{T} \dot{\mathbf{Z}} \rightarrow N S$ 
Scenario A describes evolution to $\mathrm{PNe}$ in wide systems. Their components evolve like single stars. Because in our model in most systems the secondaries have $M_{20}<0.8 M_{\odot}$, only their primaries produce PNe. Heavy mass loss which precedes the PN formation lasts for $\Delta t \sim 10^{5} \mathrm{yr}$ (e.g. Vassiliadis \& Wood 1993). If $\Delta t$ is shorter than the orbital period one may consider ejection as instantaneous. Then, under certain conditions (e.g. Hills 1983), it unbinds the binary. We list in Table 1 such PNNi in 'disrupted' wide systems as single, despite their companions may be relatively close and nebula may surround the system.

The main scenarios for formation of $\mathrm{PNe}$ by means of CEs are given by Scenario B. Branch B.1 describes formation of single progeny of initially binary stars. The reasons for formation of CE and merger are small $A_{0}$ and $q_{0}=M_{10} / M_{20}$. Merger products may differ from initially single stars by faster rotation. However, PNe which may be produced by them, perhaps will not differ from the progeny of single stars (or components of wide binaries). Systems following B.2 have $A_{0}$ and/or $q_{0}$ higher than in B.1. This prevents merger of components after the first CE stage. Total birthrate of systems which pass through B. 2 is only $\sim 25 \%$ of the total birthrate of close systems, because primaries of close binaries with $M_{10} \lesssim 2.5 M_{\odot}$ produce 'lazy' helium WDs unable to ionize nebulae.

Post PNN+MS systems with $M_{20} \lesssim 1 M_{\odot}$ may become CVs (B.3) if components are close enough and angular momentum loss (AML) via magnetic stellar wind and/or gravitational waves radiation (GWR) may bring them into contact in Hubble time and may support, after the RLOF, dynamically stable mass exchange at a rate below the critical one. Only $\sim 1 \%$ of close binary PNNi with MS companions may be pre-CVs with CO-accretors. About 10 such systems are known (see King et al. 1994).

Systems with $M_{20} \gtrsim 0.8 M_{\odot}$, which do not produce CVs, proceed from B.2 to B.4 and experience the second $\mathrm{CE}$ stage. If components merge (B.5), a rapidly spinning giant and a single PNN may form, like in B.1. If system avoids merger, a PNN accompanied by a WD may be formed (B.6). The percentage of WD+PNN systems is low because in most systems the secondaries, due to low mass, do not become WDs at all or produce 'lazy' He WDs. When PNN cools, a double degenerate forms (WD+WD). Among known close WD+WD systems, WD 1317+453, WD 1241-010, WD $2331+290$, and WD $1101+364$ probably produced PNe in the first CE event, but their currently observed components were 'lazy' (Iben et al. 1997). Only L870-2 may have two $\mathrm{CO}$ components and followed entire route B.6. Components of WD+WD systems with $P_{\text {orb }} \lesssim 9 \mathrm{hr}$, may merge in Hubble time. The total galactic rate of mergers of $\mathrm{CO}+\mathrm{CO}$ white dwarfs is $\sim 0.005 \mathrm{yr}^{-1}$. If total mass of the system exceeds $1.4 M_{\odot}$, the outcome may be a SN Ia (branch B.7). The model rate of SNe Ia is $0.003 \mathrm{yr}^{-1}$. Thus, PNNi with sus- 


\section{L.R. Yungelson}

pected massive WD companions may be interesting as possible precursors of supernovae. We should mention, however, that calculations of merger still did not tell if it results in thermonuclear explosion of carbon. If pair is not massive enough, a single WD forms (B.8).

Scenario $\mathrm{C}$ describes a possible way to formation of hydrogen-deficient supergiants, perhaps, of $\mathrm{R} \mathrm{CrB}$ type. The primary becomes a helium WD after a steady RLOF, without ejection of a CE. Detectable nebula is produced by the secondary, which accretes enough mass for formation of a $\mathrm{CO}$ dwarf. If $\mathrm{He}$ and $\mathrm{CO}$ WDs are close enough, they may merge in Hubble time into an object with $\mathrm{CO}$ core and extended He-rich envelope. If $\mathrm{R} \mathrm{CrB}$ stars end their evolution by ejection of the helium envelope, like hydrogenrich AGB stars, they may produce hydrogen-deficient PNe. Despite low incidence of this scenario $\left(\sim 0.001 \mathrm{yr}^{-1}\right)$, it may produce a considerable proportion of all observed hydrogen-deficient (super)giants because merger products have an order of magnitude larger reservoir of fuel than 'final flash' products (Iben et al. 1996).

In scenario $\mathrm{D}$, the primary with $5 \lesssim M_{1} / M_{\odot} \lesssim 10$ produces the first $\mathrm{CE}$. The remnant of the primary is, in most cases, a 'lazy' helium star. The remnant expands after exhaustion of $\mathrm{He}$ in the core (e.g. Iben \& Tutukov $1985)$ and may produce the second (hydrogen-deficient) CE. Carbon-oxygen core of the primary becomes a PNN and, later, a non-DA white dwarf. For nonmerging systems (D.1) further evolution is similar to the branch B.2. If components merge inside CE (D.2) one may expect formation of a giant, which later produces a $\mathrm{PN}$ and becomes a WD.

Scenario E describes formation of hypothetical TŻOs (Thorne \& Żytkow 1975). Our analysis shows that NS may merge with CO WDs at a rate comparable with the rate of mergers with low-mass MS stars $\left(\sim 10^{-4} \mathrm{yr}^{-1}\right)$. Initial primary has $M_{10} \gtrsim 11.5 M_{\odot}$ and becomes a NS after a SN Ib-type explosion. The secondary with $5 \lesssim M_{20} / M_{\odot} \lesssim 10$ becomes a nucleus of a hydrogen-deficient PN after the stage of a helium star, like in D.1. As a consequence of three $\mathrm{CE}$ stages, a low-mass binary pulsar may arise (at the NS+WD stage). Later, close enough pairs may merge into TŻOs because of AML via GWR. One may expect that $\mathrm{CO}$ envelopes of these TŻOs rapidly collapse like in objects with low mass hydrogen envelopes (Podsiadlowski et al. 1995). A single recycled NS may appear.

In scenarii $\mathrm{A}$ and B.4, between two $\mathrm{PN}$ stages, detached WD plus giant systems may manifest themselves as symbiotic stars (SyS). These two main channels of formation of SyS with CO white dwarf accretors have incidence rate of $0.04 \mathrm{yr}^{-1}$ (Yungelson et al. 1995).

Figure 1 shows the distribution of model binary $\mathrm{PNNi}$, over the orbital periods. The sample is totally dominated by PNNi with MS companions. The distribution of $\mathrm{PNNi}$ weighted over the geometrical probability 
of eclipses $2 R_{2} / A$ shows that only the shortest $P_{\text {orb }}$ systems with MS companions may be discovered as eclipsing ones.
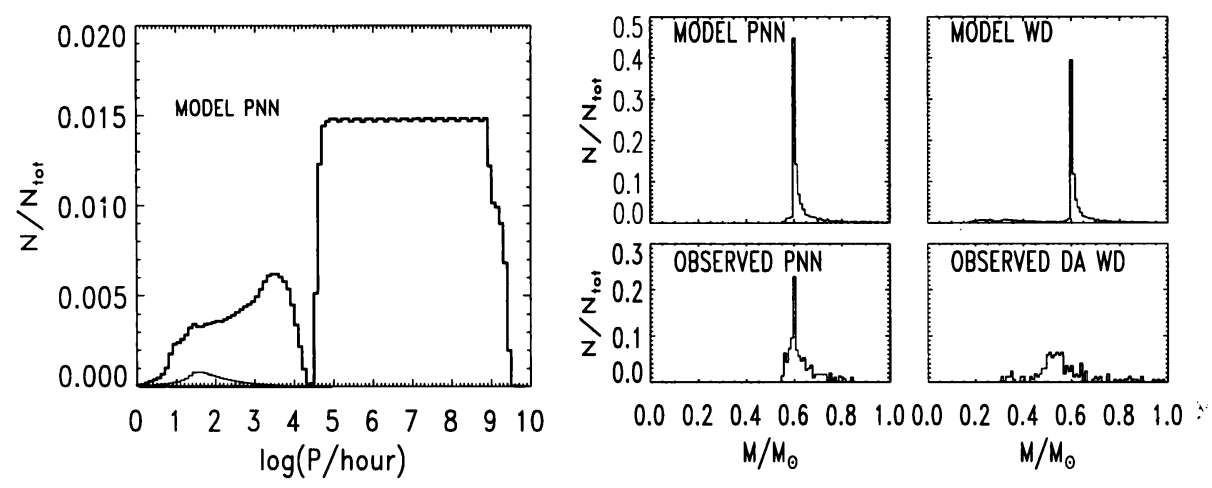

Figure 1. Left - distribution of model sample of PNNi over orbital periods. Numbers in $\Delta \log P=0.1$ wide bins are normalized by total number of systems. Lower line shows distribution weighted over geometrical probability of eclipses. Right - distribution of model and observed samples of PNNi and WDs over masses. The numbers in $\Delta M=0.01$ wide bins are normalized by total number of objects in each sample.

Figure 1 gives also the model distribution of PNNi over mass and the observational one based on infrared and radio data (Zhang \& Kwok 1993) ${ }^{1}$. Close binary PNNi influence the spectrum in two ways. Abortion of evolution before the end of AGB phase generates low-mass nuclei. Mergers of components in close binaries generate excess of high mass (hence, more luminous) nuclei. Both effects make the peak in the distribution at $\sim 0.6 M_{\odot}$ less pronounced than one would expect for single stars only (or wide binaries). The same is true for the spectrum of WDs (see below). It's premature to require a quantitative agreement between the model and observations, because of huge uncertainties in both of them. Both distributions have sharp peaks at $\sim 0.6 M_{\odot}$ and higher mass tails. Model distribution, at masses below $0.6 M_{\odot}$, falls more sharply than the observed one. The latter does not contain any objects less massive than $0.55 M_{\odot}$, supporting the inference about the 'lazyness' of overwhelming majority of helium WDs.

A natural class of objects for comparison with PNNi are WDs. In Fig. 1 we plotted the mass spectra of our model sample of 'detectable' WDs (Iben et al. 1997) and of combined sample of $164 \mathrm{DA}$ white dwarfs from Bergeron et al. (1992) and Bragaglia et al. (BRB, 1995) ${ }^{1}$. The mass spectrum of observed PNNi peaks at higher masses than that of DA type WDs. Observed WDs have a much larger spread in mass than our theoretical sample. This may reflect uncertainties in conversion of surface gravities and temperatures

\footnotetext{
${ }^{1}$ We have used $0.01 M_{\odot}$ binning suggested by the number of significant digits in observational estimates.
} 


\section{L.R. Yungelson}

into masses [see discussion in Zhang \& Kwok (1993) and BRB]. Mass spectrum of WDs which descend from close binaries, like for PNNi, is different from that of the progeny of single stars (or components of wide binaries). The most important difference is presence of low-mass $\left(M \lesssim 0.5 M_{\odot}\right)$ helium WDs. However, because most of WDs and PNNi descend from the same wide binaries, the spectra of observational samples must, at least, to peak at the same mass. This is not the case. Almost certainly, the accuracy of 'observational' masses is overestimated.

The birthrates of PNe and WDs have not to be equal. In close systems the birthrate of WDs, for our assumed CE parameter $\alpha_{c e}=1$ and slopes of IMF and mass-ratio distribution, is $\sim 0.48 \mathrm{yr}^{-1}$, by $\sim 20 \%$ higher than the birthrate of close systems themselves and more than twice as high as the birthrate of PNNi. The reasons are evident: most helium WDs are unable ionize the remainders of CEs and some close binaries produce lowmass helium stars which do not produce $\mathrm{PNe}$. But some systems produce two PNe. As a result, total birthrate of $\mathrm{PNe}$ is $0.75 \mathrm{yr}^{-1}$, while that of WDs - $0.97 \mathrm{yr}^{-1}$. We have to mention that because of presence of bright companions only $\sim 70 \%$ of young WDs are available for observations.

To summarize, population synthesis model, which accounts for production of PNe via ejection of matter by AGB stars and of CEs by close binaries, gives the birthrate of $\mathrm{PNe}$ consistent with the estimate of their galactic incidence based on local volume density (Peimbert 1983). About $1 \%$ of galactic PNNi may have companions which in later evolutionary stages merge with them giving rise to certain spectacular astrophysical objects.

Acknowledgements. L.Y. acknowledges support by IAU travel grant.

\section{References}

Bergeron, P., Saffer, R.A., Liebert, J., 1992, ApJ, 394, 228

Bragaglia, A., Renzini, A., Bergeron, P., 1995, ApJ, 443, 735

Han, Z., Podsiadlowski, Ph., Eggleton, P. P., 1995, MNRAS, 800

Hills, J. G., 1983, ApJ, 267, 322

Iben, I. Jr.,Tutukov, A. V., 1985, ApJSS, 58, 661

Iben, I. Jr., Tutukov, A. V., 1993, ApJ, 418, 343

Iben, I. Jr., Tutukov, A. V., Yungelson, L. R., 1996, ApJ, 456, 750

Iben, I. Jr., Tutukov, A. V., Yungelson, L. R., 1997, ApJ, January 20 issue

King, A. et al., 1994, MNRAS, 269, 907

de Kool, M., 1990, ApJ, 358, 189

Peimbert, M. 1993, in Planetary Nebulae, eds. R. Weinberger and A. Acker (Dordrecht: Kluwer), 523

Podsiadlowski, Ph., Cannon, R. C., Rees, M. J., 1995, MNRAS, 274, 485

Thorne, K., Żytkow, A., 1975, ApJ, 199, L19

Vassiliadis, E., Wood, P., 1993, ApJ, 413, 641

Yungelson, L. R., Tutukov, A. V., Livio, M., 1993, ApJ, 418, 794

Yungelson, L. R. et al., 1995, ApJ, 447, 656

Yungelson, L. R. et al. 1996, ApJ, 466, 890

Zhang, C. Y., Kwok, S., 1993, ApJSS, 88, 137 